\title{
«Tu m'entends, inutile!» \\ Expression populaire de la rage féminine envers les hommes dans l'œuvre chantée de Paquita la del Barrio ${ }^{i}$
}

\author{
Guitté Hartog et Jacqueline Chénard
}

\begin{abstract}
In Mexico a popular singer expresses - for millions of women - taboo feelings such as rage, shame and frustration left by the experience of disappointed love. Far from the political correctness belonging to the traditional feminine ideal or that of women's emancipation, this is a true cultural transgression, contrasting with the «proper» way Latino-American women and many others ought to accept having been deceived, abandoned, poorly loved, poorly made love to, now alone, often with the children of these men from whom they expected everlasting happiness, passion, support and tenderness.
\end{abstract}

Je ne sais pas ce que j'ai de travers contre les hommes

Pourquoi diable, je les hais autant

Et comment, je ne vais pas les haïr?

S'ils sont la cause d'autant de pleurs

Mon grand-père trompait ma grand-mère

Mon père maltraitait ma mère

Et ce fils de... continue dans la même lignée...

Tu m'entends, inutile...

Oh ! Hommes «malfrats»!

Là, on vous chante....

Ou vous vous replacez,

$\mathrm{Ou}$ on vous castre

Vous entendez, inutiles!

Ce qui peut vous arriver!

(Extrait de la chanson «Hombre malvados», Traduction libre)

Au Mexique, La Paquita, une femme au physique imposant, vêtue de la tête aux pieds de paillettes, chante avec beaucoup de glamour son désenchantement pour ce qu'elle appelle Le club des inutiles, c'est-à-dire les mâles qui ont trompé leurs femmes, leur ont menti, furent piètres amants et se sont fait entretenir par les femmes qui les ont aimés. Le spectacle retient d'autant plus l'attention qu'en terre 
latino-américaine, le machisme et l'abnégation féminine font partie de l'identité nationale.

En tant que dame,

Je devrais me taire.

Mais maintenant, tout le monde va savoir

Que t'es un désastre au lit...

(Extrait de la chanson «Taco placero»

Traduction libre )

Dans un pays où on enseigne aux fillettes qu'elles sont plus mignonnes quand elles se taisent, (dicton populaire) ${ }^{\mathrm{ii}}$; soudainement se déploie un choeur de milliers de femmes de tous âges qui chantent à s'époumoner leur mépris pour les hommes qui les ont déçues, dirigé par une chanteuse qui revit à chacun de ses spectacles, émue jusqu'aux larmes, la rage que lui ont laissée ses déceptions amoureuses.

Comme chien tu m'as suivi

Comme chien tu me poursuivais...

À ma porte tu grattais...

Pour recevoir mon amour...

Une fois que tu l'as eu

Tu fus un homme rude et cruel

Tu fus un chien traître

Capable de mordre la main qui te nourrissait

De chien à chien

Qui est le plus chien et le plus canaille?

Toi qui as pu mordre la main qui prenait soin de toi.

Je viens te demander pardon

Pour te comparer avec lui

Pardon...

Pardonne-moi cher ami

Que me pardonne ton chien

Pour le comparer à toi

Tu m'entends inutile!

Je suis en train de parler au chien!

Que me pardonne ton chien

Pour l'avoir comparé à toi

(Extrait de la chanson «Que me perdone tu perro»,

Traduction libre) 
Malgré quelques décennies de féminisme et de liberté d'expression, les manifestations de la colère féminine demeurent un tabou important. Les femmes qui manifestent de la rage sont socialement perçues comme hystériques ou folles à lier. Le féminisme a voulu les femmes indépendantes et fortes, ce qui a laissé peu d'espace pour parler des sentiments et des besoins amoureux. Trop longtemps confinées à la sphère privée, les femmes ont dû lutter pour se faire une place dans ce qu'on considérait l'univers des hommes, c'est-à-dire le monde professionnel et politique. Après une si longue répression intellectuelle et sociale, il n'était pas dans les priorités des féministes de se laisser aller dans le sentimentalisme et l'émotivité. La colère des femmes fut sublimée, dans le meilleur des cas, dans des luttes pour une meilleure justice sociale, et dans le pire des cas, intellectualisée dans un discours théorique qui faisait des hommes les bourreaux et les oppresseurs des femmes depuis la nuit des temps. En effet, les luttes féministes ont permis aux femmes de réclamer aux hommes de partager les taches domestiques et le soin des enfants et de divorcer des hommes, mais pas nécessairement d'exprimer leur colère, leur tristesse et leur déception de ne pas rencontrer des compagnons à la hauteur de leurs aspirations amoureuses.

\author{
Tu m'as tant déçue \\ Que je t'envoie un chèque en blanc \\ $\grave{A}$ ton nom, juste pour toi \\ C'est pour la quantité que tu veux \\ Où ça dit mépris, ça doit être ton prix \\ Et ça va signé par moi
}

(Extrait de la chanson «Cheque en blanco», Traduction libre)

Quant à l'œuvre qu'interprète «La Paquita», elle permet justement de rendre publique une partie importante et douloureuse de la vie privée des femmes. Sans faire de politique, à grands coups de chansons et d'insultes, elle met des mots sur des situations et des émotions que de nombreuses femmes refoulent parce que considérées honteuses. En effet, ses prestations mettent en lumière la cause de beaucoup de dépressions, d'insomnies, de maux de tête et de crises de boulimie qu'expérimentent de nombreuses de femmes qui ne furent jamais aimées par un homme comme elles l'auraient souhaité, qui se rendent compte que leur vie amoureuse fut un désastre, qu'elles ont aimé des hommes qui n'en valaient pas la peine, et eu des enfants d'hommes «inutiles», irresponsables, qui pour comble n'étaient pas de très bons amants. Son discours n'a rien d'intellectuel, il vole même plutôt bas, mais il n'a d'autre fonction que de libérer les rêves frustrés des nombreuses femmes qui se meurent d'envie de partager leur vie avec un compagnon qui soit leur égal et non un de ces «inutiles». 
Si à dormir tu m'as amenée dans ton lit T'aurais dû me le dire...

Pour dormir j'ai mon propre lit

Et plus grand et plus chaud...

Toute la nuit, je l'ai passée à t'attendre

En rêvant toute seule

Pendant que tu ronflais

Pauvre petit pistolet

Tu ne tires rien

$\mathrm{Ni}$ de temps en autres

Tu m'entends inutile!

Quelle ingrate fut la nature avec toi!

(Extrait de la chanson «Pobre pistolita», traduction libre)

Pour les femmes latino-américaines que trop souvent on a convaincues que tous les hommes sont pareils et congénitalement incapables de fidélité, les chants de Paquita la del Barrio acquièrent des vertus thérapeutiques, une sorte de remède au fatalisme. D'une certaine façon, la brutalité du répertoire que chante La Paquita fait sourire, rassure les femmes sur la légitimité de leur colère et les soulage de sentir qu'elles ne sont pas les seules à vivre pareilles situations. Bien sûr, le ton est à la vengeance et elle frappe «où ça fait mal» c'est-à-dire directement dans l'orgueil du macho, en insultant sa «Sainte-Mère», en le traitant d'impuissant et de cocu.

Moi, qui connement t'avais toujours été fidèle

Aujourd'hui ce fut différent...

Trois fois je t'ai trompé

Trois fois je t'ai trompé

Trois fois je t'ai trompé

La première fois par colère

La seconde par caprice

La troisième fois par plaisir 


\title{
Trois fois je t'ai trompé Trois fois je t'ai trompé Trois fois je t'ai trompé \\ Et, après ces trois fois $\mathrm{Et}$, après ces trois fois Je ne veux plus rien savoir de te revoir.
}

(Extrait de la chanson à succès «Tres veces te engañé», Traduction libre)

Son succès populaire montre la vitalité de l'inspiration que soulèvent les passions amoureuses. Seul l'amour démesuré peut pousser un Jacques Brel à supplier une femme de le laisser être l'ombre de son chien ${ }^{\text {iii }}$ et une femme comme Paquita à implorer un chien de lui pardonner de l'avoir comparé à son ex-amant. Ces comparaisons montrent bien que les relations qui unissent les hommes et les femmes, et qui les séparent, comportent une grande part d'irrationalité et de pathos, surtout quand elles se traduisent en chansons. Mais au bout du compte, avec quelques adaptations mineures au texte, toutes les chansons qu'interprètent Paquita, Brel et tous les autres pourraient facilement être interprétées d'une façon crédible autant par un homme que par une femme. Et dans le cas de Paquita, il n'y a aucun doute sur la sincérité et la puissance des sentiments exprimés. L'assistance y croit... suffisamment pour qu'on voie des femmes se lever, chanter à gorge déployée, les bras grands ouverts; et les hommes détourner la tête et rougir comme s'ils venaient de recevoir une gifle en écoutant La Paquita, accompagnée de son choeur de femmes, déclamer son grand tube...

\author{
Rat immonde \\ Animal rampant \\ Crasse de la vie \\ Déchet de la vie \\ Je te hais et je te méprise \\ Rat à deux pattes \\ C'est à toi que je parle
}

Tu m'entends, inutile !

Maudite sangsue

Maudite coquerelle 
Rat à deux pattes...

C'est à toi que je parle

Parce qu'une bestiole rampante

Même en restant la plus maudite

Comparée a toi

Reste bien modeste...

(Extrait de la chanson «Ratas de dos patas», Traduction libre)

La charge émotive que porte la voix de Paquita la del Barrio, qui ne mâche pas ses mots, permet d'exprimer la douleur refoulée par les femmes ébranlées à la suite d'une déception amoureuse. Il s'agit d'une véritable transgression culturelle qui contraste avec la façon «convenable» dont les femmes latino-américaines et bien d'autres devraient accepter d'avoir été trompées, abandonnées, mal aimées, mal baisées, d'être maintenant seules, bien souvent, avec les enfants de ces hommes de qui elles attendaient le bonheur, la passion, l'appui et la tendresse pour toujours. Il ne s'agit pas d'une solution et encore moins d'une proposition pour construire des relations harmonieuses entre les hommes et les femmes, il s'agit plutôt d'un défoulement cathartique, d'une ordonnance, d'un antibiotique, d'un prix de consolation bon marché pour garder le goût de chanter, de célébrer la vie qui continue malgré tous ces «inutiles» qui leur ont tordu le cœur.

\section{Notes}

i Paquita la del Barrio «celle du quartier» est une chanteuse populaire du Mexique qui, comme son surnom l'indique, se veut du peuple. Les auteurs des chansons ne sont pas connus, ni identifiés. "Tu m'entends inutile!» constitue sa phrase phare. La légende veut qu'elle fut inspirée de son premier mari, père de ses trois enfants et lancée en plein spectacle lorsque la Paquita s'est soudainement rendu compte que «son homme» assistait à son spectacle au bras de sa nouvelle conquête.

ii Traduction libre du dicton populaire «Calladita te vez más bonita».

iii Extrait de la chanson populaire «Ne me quitte pas». 